\title{
Endurance tests of single machines production
}

\author{
Valery Kasyanov ${ }^{1}$, Viktor Deryushev ${ }^{1}$, Leonid Shulkin ${ }^{1}$, Evgeny Kosenko ${ }^{1, *}$, and Anastasia \\ Kotesova $^{1}$ \\ ${ }^{1}$ Don State Technical University, The Faculty of Road-Transport, Russia
}

\begin{abstract}
The paper proposes to consider the reliability of machines in terms of mass and individual production. The proposed approach makes it possible to assess the impact of various failures of machine parts on the reliability of single-purpose vehicles. Failures occurring as a result of fatigue wear are considered, but they are of a sudden nature of failure. A condition is defined to prevent such failures, from which the assigned resource to the limit state of the part must be less than the resource of each particular part that may be susceptible to sudden failure. This condition can be applied both for serial production machines and for single-source machines.An algorithm for determining sample and aggregate parameters and an algorithm for resource testing for reliability for single-source machines are proposed. The key stage for the development of the algorithm is the resource testing of machines, the carrying out of which makes it possible to objectively assess the reliability of the loaded parts. To reduce the period for obtaining test results, it is necessary to carry out accelerated resource tests of machines, which can be achieved by intensive operation of machines with short stops for inspection and maintenance.Failures resulting from tests constitute a sample that, after being processed by theoretical laws, allows the determination of the smallest values. Studies have shown that even with the estimation of sample values, the possibility of premature failure continues. In this regard, the paper proposes to use the aggregate parameters to estimate the minimum values. The use of such an algorithm will make it possible to exclude the possibility of the onset of premature failures by determining the period of operation of vehicles during which the failure-free operation of cars is guaranteed.
\end{abstract}

\section{Introduction}

Endurance testing takes an important place in the system of ensuring the reliability of oneoff machines production [1-4].

Before testing the entire composition of the machine parts should be divided, taking into account the effects of the parts failure on the machine failure (see table).

\footnotetext{
*Corresponding author: a123lok@mail.ru
} 
Table 1. Influence of damage types to parts on the failure free operation of the machine (unit)

\begin{tabular}{|c|c|c|}
\hline $\begin{array}{c}\text { Parts with a } \\
\text { sudden failure }\end{array}$ & Parts with a gradual failure & $\begin{array}{c}\text { Unloaded } \\
\text { the details }\end{array}$ \\
\hline Fatigue & Wear and fatigue & No refusals \\
\hline \multirow{2}{*}{ Not diagnosed } & $\begin{array}{c}\text { Diagnosed, failures are } \\
\text { prevented by planned substitutions }\end{array}$ & \\
\cline { 2 - 3 } & \multicolumn{2}{|c|}{ Do not cause machine failure } \\
\hline
\end{tabular}

From the parts failures consideration it is visible that the machine (unit) failure free operation is influenced by sudden fatigue parts failure. To prevent such failures it is necessary to fulfill the conditions $[5,6]$ :

$$
T_{\text {arp }}<T_{\text {rpiMIN }}
$$

where $\mathrm{T}_{\text {arp }}$ - is the assigned resource to the limit state of the part,

$\mathrm{T}_{\mathrm{rpi}}$ - is the $\mathrm{i}$-th part resource.

Therefore, proceeding from this condition it is advisable in addition to the main machine to have a backup with the same operation type.

With respect to the serial machine indicators for its reliability properties are used both for the machine as a whole and for units and parts [7,8]. In addition, complex indicators are used: availability and technical use factors $[9,10]$. The same indices are also determined for a one-off machine production.

Development of algorithms for resource reliability testing

The main parameter of the TRDI resource detail depends on the strength, loading, factors increasing or decreasing strength and acting stresses parameters, etc.

$$
T_{p g i}=f\left(\sigma_{-1}, \sigma_{a}, \beta, \kappa,\right),
$$

where $\sigma_{-1 \mathrm{~g}}$ is the limit of parts endurance

$\sigma a$ is the component strength increase coefficient

$\kappa$ is the effective voltage increase coefficient.

This calculated resource with an allowable error is necessary to be related to each object of the assembly or to its sample from (Fig. 1), [5,10,11].

Thus, different parts (shafts, gears, chains, etc.) with the same resource will form a homogeneous collection and its sample.

At the same time the minimum part resource should be used as the main parameter.

Then the sample (assembly) homogeneity of the will be determined by the same minimal details resources $\mathrm{T}_{\text {pimin }}$

$$
T_{\text {plmin }} \approx T_{p 2 \min } \approx \ldots \approx T_{\text {pnmin }} \approx T_{\text {rgd }}
$$

where $\mathrm{T}_{\mathrm{rgd}}$ is the given resource up to a limiting condition of a detail. 


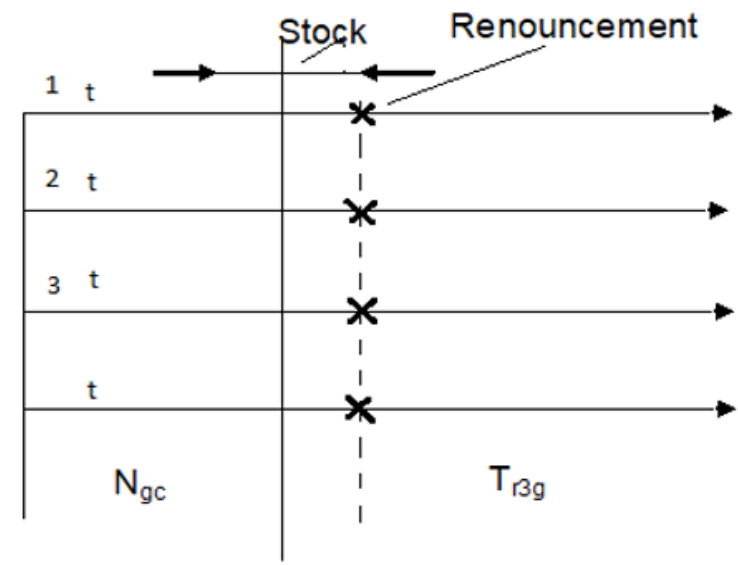

Fig. 1. The work and failure scheme of identical details in the main parameter Tpgi, but different in purpose

The main condition for ensuring the same main parameters of details with sudden failures of the Tpgi resource (machines of single production) is a calculation using the Veler-Serensen-Kogayev formula based on the initial statistics of fatigue strength and loading.

At the presence of probabilistic distributed sampling parameters $\sigma-1, \sigma a, \beta, \kappa$ and others, modeling is carried out with respect to the dependence (1) with a further approach to the distribution of the aggregate and then to finding its minimum value for the required parameters (Figure 2) (12-13) .

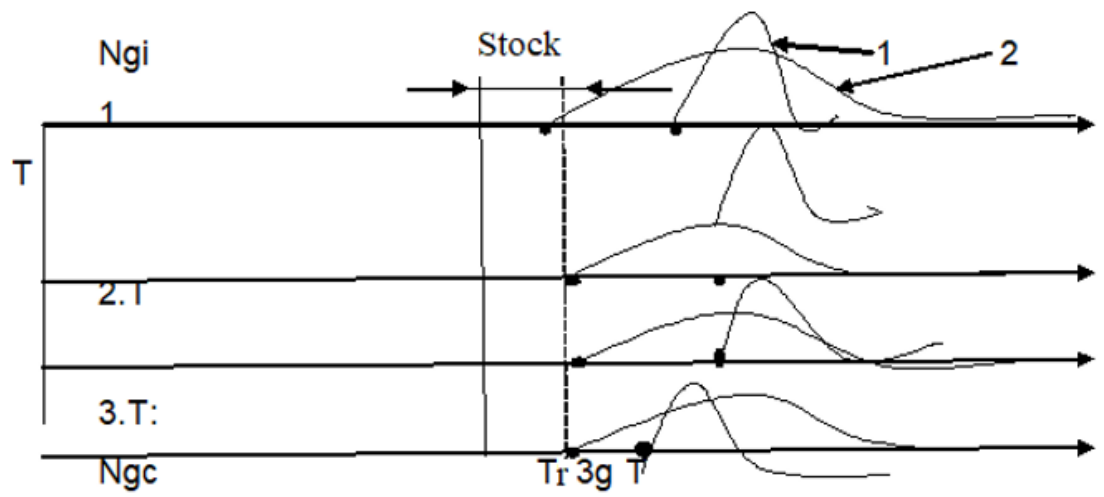

Fig. 2. Probabilistic distributions of the samples resource and sets of parts with fatigue sudden failures: 1-sample; 2-assembly.

Information for calculating the detail resource is available in Figure 3. 


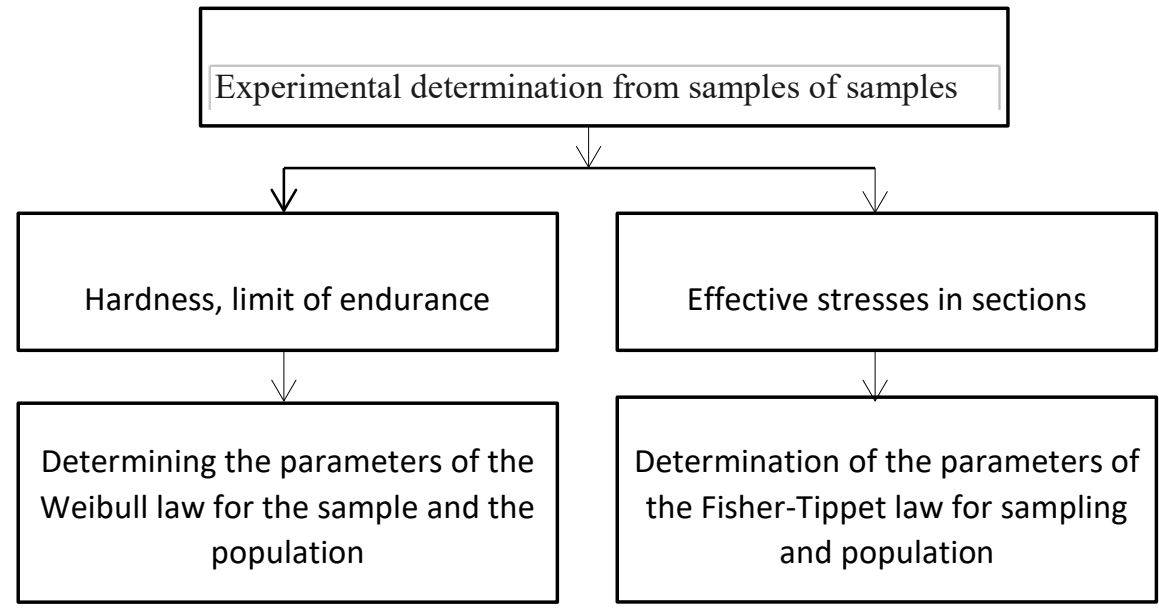

Fig. 3. Information for calculating detail resource.

The determination of the parameters of the sample and assembly distribution is given in Figure 4.



Fig. 4. Determining the parameters of the sample distribution and the assembly.

Modeling is advisable to make not for each detail of the assembly, but for sample n6 = 10-100 details. Having determined the parameters of the Weibull distribution for the selection of details, it is necessary to proceed to the calculation of the Weibull distribution parameters for the assembly. For cases of different amplitude (Figure 5). 


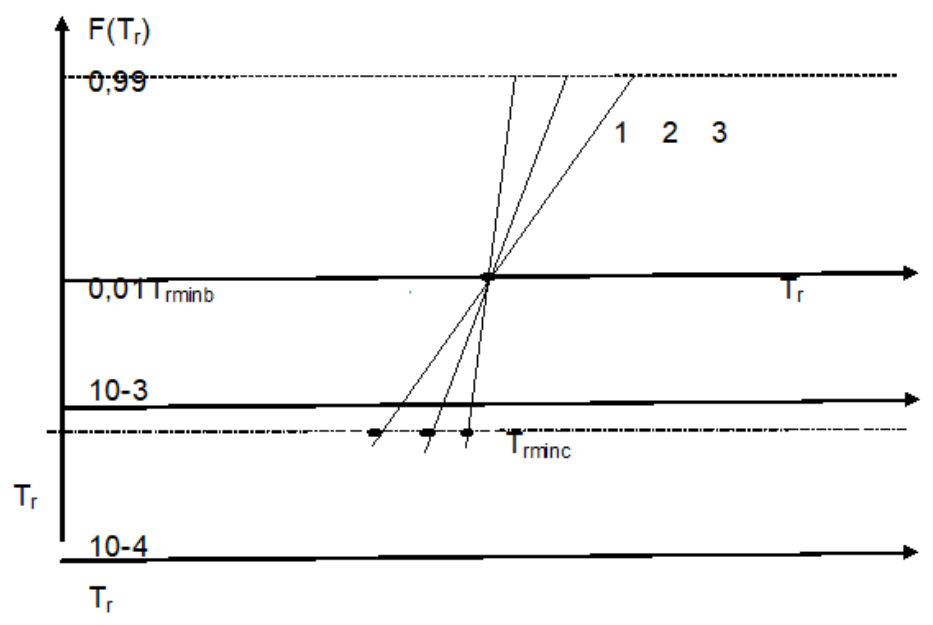

Fig. 5. Distribution of Weibulla details with different amplitude: $1-\mathrm{R}=30 ; 2-\mathrm{R}=50 ; 3-\mathrm{R}=70$.

Gradual failures are eliminated. Sudden failures are excluded to Tp3 by scheduled substitutions or by requests for repairs and should not occur earlier than Tg3

Primary machine 1 , standby machine 2 (Figure 6) [11, 12].
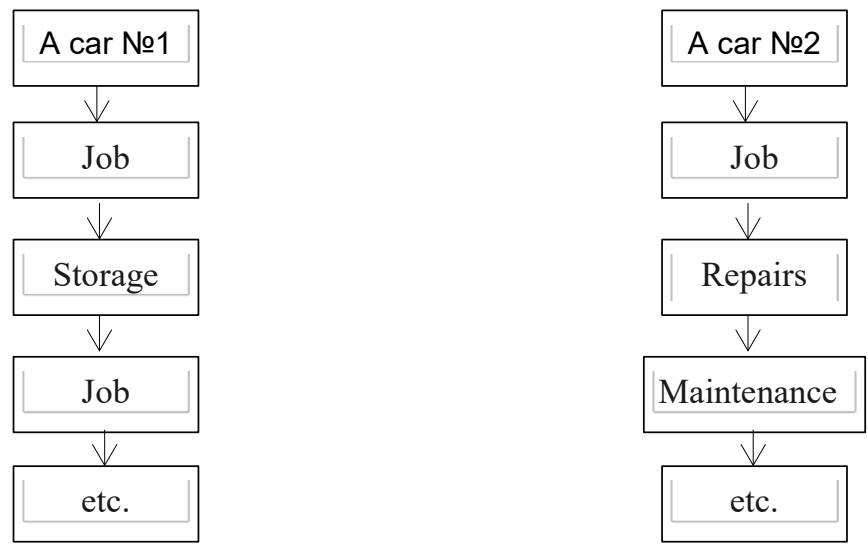

Fig. 6. Ensuring the reliability of the primary and standby machines.

Thus, ensuring the reliability of single production machines when at first glance there is no possibility of using the acquired probabilistic-static and other methods of ensuring absolute safety (responsible machines) of serial machines, there is also a real opportunity to solve this problem.

The analysis of various types of tests showed that the most effective type is the machine (unit) operation, where almost every part is being tested and simultaneously serving as a loading element for another adjacent part.

Further it is necessary to obtain the sampling and aggregation parameters (Figure 7) [11, $12]$. 


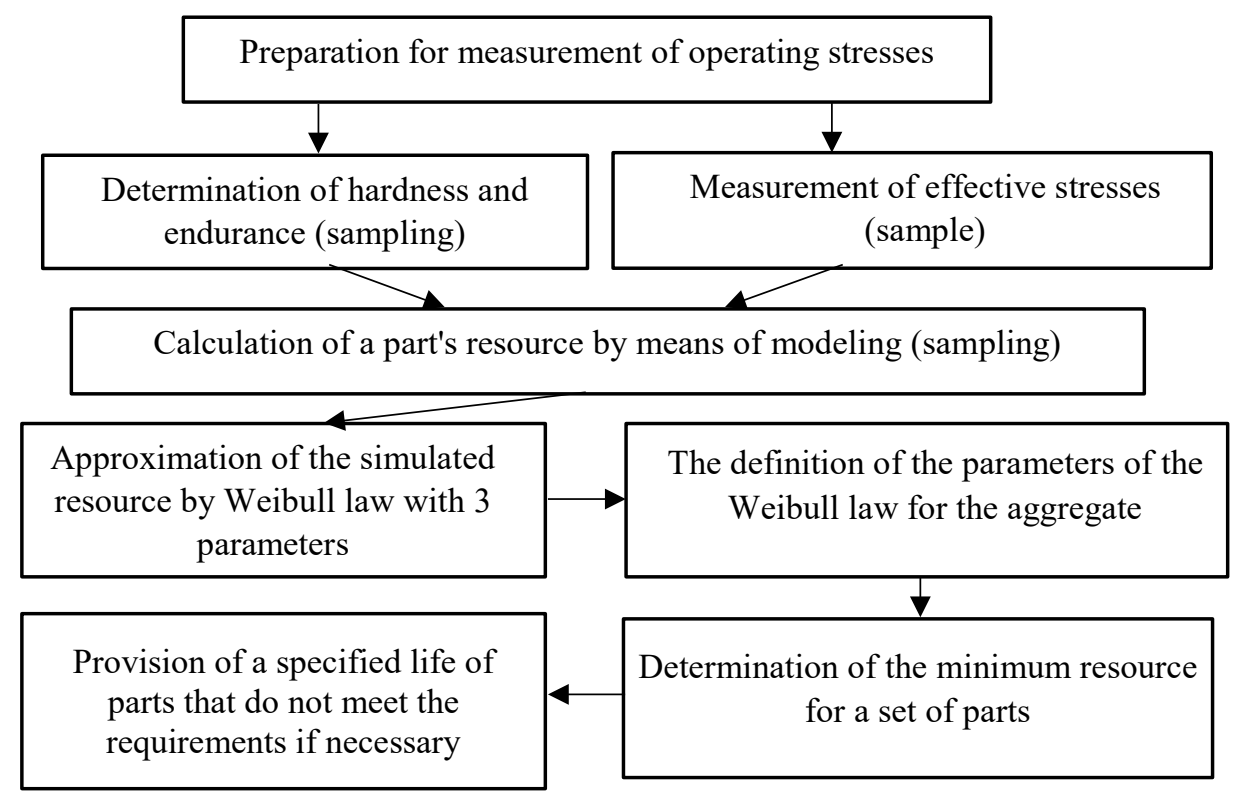

Fig. 7. Algorithm for obtaining the sampling and aggregation parameters.

Thus, the machine (unit) turns into a self-stand. If any part fails it must be replaced and the machine should be continued to operate. The algorithm of resource tests of the two cars is presented in Figure 8.

Since the requirements for the service life of a number of machines have increased accordingly to 20 thousand hours and 20 years, the actual task is to carry out accelerated tests of machines for experimental confirmation of estimated resources and service lives of machines and their components. The experience of the Japanese company Komatsu for the production of construction machines is known, referring to 1970-1980. The selection of bulldozers from 32 machines for earthmoving was used for 20 - 22 hours a day and for a year the machines had been working out for about 250 days or $20 * 250=5000$ hours. Consequently, a given resource of 10 thousand hours has been worked out within 2 years at a standard of 10 years, i.e. acceleration in time with an acceleration factor of 5 during the tests.

In case of increased demands on the machine for a resource of up to 20 thousand hours and a service life of 20 years, it is required to increase the intensity of work up to 23 hours a day and up to 300 days a year. Then the machine will work for 20 years $20 * 300=6000$ days and about $20,000 / 6000=3.3$ hours per day. 


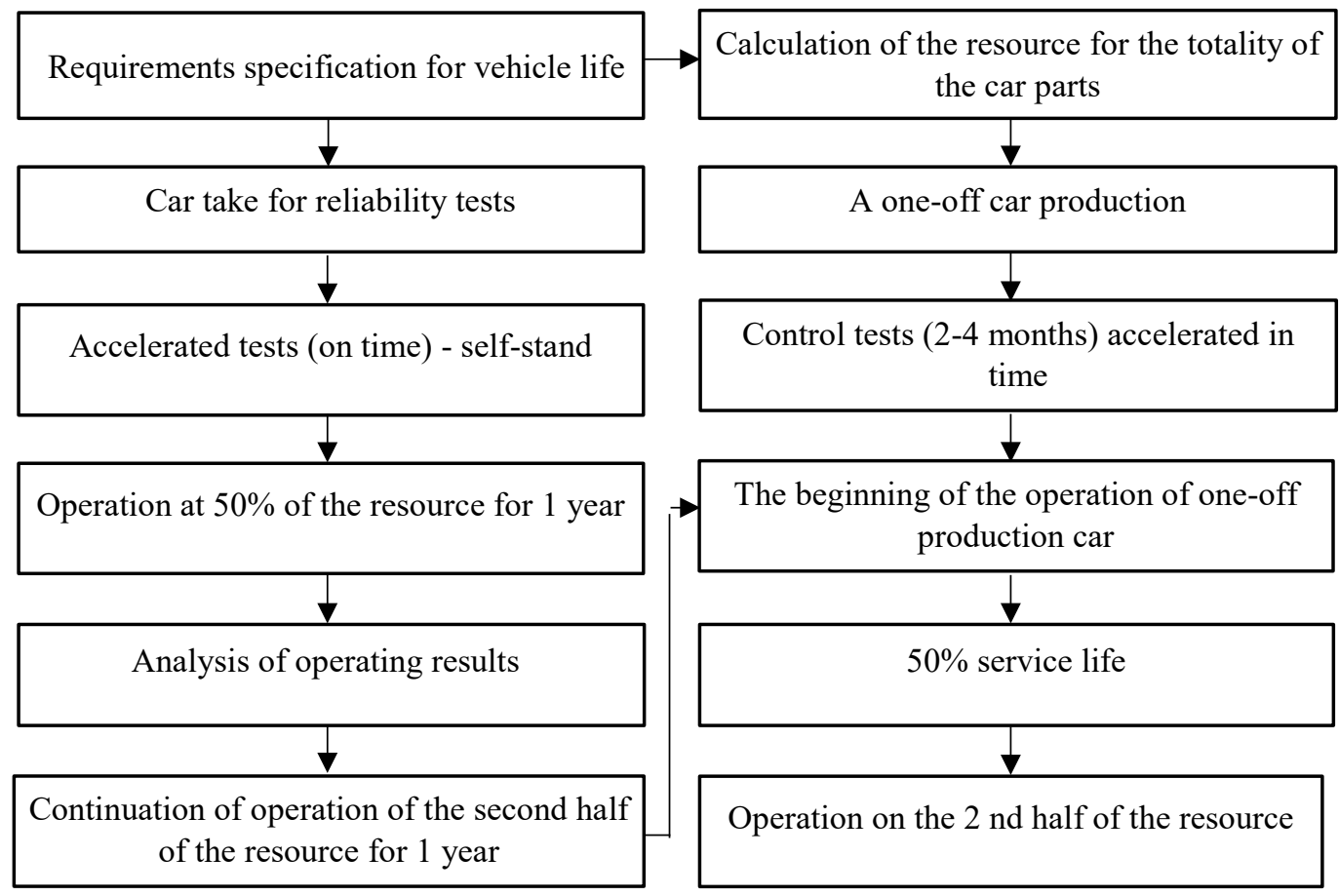

Fig. 8. Algorithm of resource tests for the reliability of two cars.

If the machine is used for 23 hours a day, the acceleration factor will be $23 / 3,3 \approx 7$, and the test duration will decrease to $20 / 7 \approx 2,9$ years instead of 20 years.

\section{Conclusions}

The algorithm for determining sample and aggregate parameters and the algorithm of reliability resource tests for one-off production machines have been proposed.

The algorithm of time-accelerated reliability tests with acceleration factor 7 is considered.

\section{References}

1. GOST 27.002 - 2015. Reliability in engineering. The terms of the definition. Rosstandart, p. 23.

2. V.E. Kasyanov, Tests of excavators in operation and calculation of their reliability indices on a digital computer, Reliability and quality control, №6, p.15-19 (1976).

3. GOST 27401-84 (STSEV 4492-84) Reliability in engineering. The order and methods of control of reliability indicators established in normative and technical documentation. General requirements. - (D.M. Belenky, V.E. Kasyanov, V.F. Kurochkin and others). M., Publishing House of Standards, 1984.

4. GOST 27-503-81 (ST SEV 2836-81). Reliability in technology. The system of information collection and processing. Methods for assessing reliability indicators (V.E. Kasyanov, A.I. Kubarev, D.M. Belenky and others). M .: Publishing house of standards. - 1981 
5. P-50-109-89. Reliability in technology. Ensuring the reliability of products. General requirements. (Bolotin V.V., Kasyanov V.E., etc.) (M.: Publishing house of standards, 1989, 15)

6. V.E. Kasyanov, Principles for creating practically trouble-free machines, Standards and quality, № 7 p. 39-42 (1988).

7. V.E. Kasyanov, T.N. Rogovenko, L.P. Shchulkin, Bases of the theory and practice of creation of reliable cars, the Bulletin of mechanical engineering, №10, p.3-6 (2003).

8. V.E. Kasyanov, A method for estimating failure-free reliability for a sample and a collection of a finite volume, Scientific Review, №11 (3), P. 785 - 788 (2014).

9. V.E. Kasyanov, L.P. Shchulkin, Determination of the gamma-percentage resource of the boom of a single-bucket excavator, Nuchnoe obozrenie, No. 10-2, P. 437-440 (2014).

10. V.E.Kasyanov, L.P. Shchulkin, Determination of the maximum loading of parts by means of modelling, Nuchnoe obozrenie, № 10-3, P. 671 - 674 (2014).

11. V.E. Kasyanov, L.P. Shchulkin, The effect of the volume of the aggregate on the strength and loading of the parts, Nuchnoe obozrenie, № 11-3, P. 782 - 784 (2014).

12. V.V. Deryushev, V.V. Bendyukov, A.I. Kostoglotov, Y.I. Yunak, A.A. Nedoluzhko, Criterial fracture analysis of cylindrical shell structures loaded by an internal pressure under a localized thermal shock, Problems of strength, № 5. P. 51-57 (1997). 